\title{
Involved Node Radiation Therapy
}

National Cancer Institute

\section{Source}

National Cancer Institute. Involved Node Radiation Therapy. NCI Thesaurus. Code

C113813.

Radiation treatment delivered only to the lymph nodes originally involved, used primarily in the treatment of Hodgkin lymphoma. 